Check for updates

Cite this: RSC Adv., 2018, 8, 11272

Received 8th December 2017 Accepted 3rd March 2018

DOI: $10.1039 / c 7 r a 13143 g$

rsc.li/rsc-advances

\section{Interface studies of well-controlled polymer bilayers and field-effect transistors prepared by a mixed-solvent method}

\author{
Fan Zhang, Yufeng Hu, (D) * Zhidong Lou, (D)* Xige Xin, Meng Zhang, Yanbing Hou (iD \\ and Feng Teng
}

The properties of semiconductor/dielectric interfaces are crucial to the performance of polymer fieldeffect transistors. The key to fabricating high-performance polymer transistors by spin-coating is solving solvent corrosion issues, wherein the solvent of the top polymer produces a rough interface or damage on the underlying polymer layer during deposition. Herein, we propose a mixed-solvent method that employs a mixture of an orthogonal solvent of the underlying polymer and a good solvent of the top polymer as the solvent of the top polymer to prepare polymer bilayers and produce a comparative study of the trap density at the semiconductor/dielectric interface of the corresponding transistor. By changing the ratio of orthogonal solvent to good solvent, namely the degree of orthogonality of the mixed solvent with respect to the underlying polymer, the interface and film qualities of polymer bilayers can be well controlled. We applied this method to spin-coat poly(3-hexylthiophene) (P3HT) on poly(methylmethacrylate) (PMMA) with a mixture of cyclohexane (orthogonal solvent) and chloroform (good solvent). The results of morphology characterizations and electrical property studies indicate the optimal ratio of cyclohexane to chloroform for preparing high-quality P3HT/PMMA bilayers for fieldeffect conduction is $7: 3$. Transistors based on the optimal bilayers with a bottom-gate/top-contact configuration and a long channel length show good performance. The trap density at the P3HT/PMMA interface is evaluated to be $3.6 \times 10^{12} \mathrm{~cm}^{-2} \mathrm{eV}^{-1}$ from the subthreshold swing, characterizing the distribution of the interface trap levels across the bandgap in P3HT. Furthermore, based on deviations from ideality in the capacitance-voltage characteristics of the metal-insulator-semiconductor capacitor in the device, the traps at the interface are found to be acceptor-type, with the trap density determined to be $2.3 \times 10^{11} \mathrm{~cm}^{-2}$. This value is in a good agreement with that estimated from the subthreshold swing.

\section{Introduction}

In polymer field-effect transistors (FETs), charge carriers are known to be transported within the first few monolayers of the semiconductor at a semiconductor/dielectric interface. The properties of this interface are of vital importance in improving charge transport and device performance. ${ }^{1-4}$ The compatibility of polymers selected as active and dielectric layers is crucial for device performance, particularly regarding solvent corrosion in solution processing, wherein the solvent for the top polymer roughens the interface or damages the lower layer during deposition., ${ }^{5,6}$ In general, to produce a polymer bilayer with a high-quality interface, a suitable solvent, in which the top polymer is highly soluble, but the underlying polymer is insoluble, must be selected to prevent damage. ${ }^{7-10}$ Such a solvent is considered perfectly orthogonal with respect to the underlying

Key Laboratory of Luminescence and Optical Information, Ministry of Education, Beijing Jiaotong University, Beijing 100044, China. E-mail: yfhu@bjtu.edu.cn; zhdlou@bjtu.edu.cn; Tel: +861051684860 polymer. The device performance of polymer FETs has been shown to be strongly correlated with the degree of orthogonality of the solvent used for the top polymer., ${ }^{7,8}$ A solvent with a low degree of orthogonality can dissolve the underlying polymer and roughen the semiconductor/dielectric interface, leading to a decrease in charge carrier mobility.

However, it is usually difficult to find perfect orthogonal solvents for polymer semiconductors or dielectrics that are soluble in common organic solvents. For example, most $\pi$ conjugated polymers and solution-processable small molecules are soluble in organic solvents that can also dissolve some practically applied dielectrics, such as poly(methyl methacrylate) (PMMA) and polystyrene (PS). ${ }^{7}$ To avoid the solvent corrosion effect, a dry transfer technique ${ }^{\mathbf{1 1}}$ has been utilized to construct polymer FETs based on poly(3-hexylthiophene) (P3HT) and PMMA with a bottom-gate/top-contact (BGTC) geometry. Recently, a P3HT-top and PMMA-bottom bilayer structure has been developed through vertical phase separation of a P3HT/PMMA blend during spin coating and used to fabricate P3HT/PMMA FETs. ${ }^{12}$ Herein, we propose a mixed-solvent 
method that employs a mixture of a perfect orthogonal solvent with respect to the underlying polymer and a good solvent for the top polymer. The degree of orthogonality of the mixed solvent with respect to the underlying polymer depends on the ratio of the orthogonal solvent to the good solvent. Accordingly, the interface morphology and roughness of the polymer bilayer can be controlled by changing the mixed solvent ratio to fabricate a high-quality bilayer. Similarly, a mixture of toluene and cyclohexane has been used to deposit poly(9,9-dioctylfluorenealt-bithiophene) (F8T2) on PMMA to fabricate F8T2/PMMA bilayer FETs with controlled interface roughness. ${ }^{10}$ This mixed-solvent method will allow the exploration and utilization of various combinations of polymer semiconductors and dielectrics to fabricate high-performance FETs that cannot be accessed through film deposition using a solution-based process due to solvent corrosion problems.

A rough semiconductor/dielectric interface is commonly thought to introduce large amounts of defects into the semiconductor layer, which enhance the formation of charge traps at the interface and hinder charge transport. ${ }^{13,14}$ Therefore, the increasing amount of interfacial traps reduces the field-effect mobility of a semiconductor. ${ }^{15,16}$ The density of interface traps can be evaluated from the subthreshold swing of an FET device. ${ }^{17,18}$ However, whether the interface traps are donor-type or acceptor-type cannot be distinguished with this method. In this regard, it is necessary to further analyze the properties of the semiconductor/dielectric interface using a capacitance technique. ${ }^{19,20}$ A polymer FET with a dielectric layer sandwiched between a gate electrode and a semiconducting layer can be considered a metal-insulator-semiconductor (MIS) capacitor. Capacitance-voltage $(C-V)$ measurements of an MIS capacitor provide a useful approach for studying the properties of a semiconductor/dielectric interface. This technique can rapidly evaluate the total interface-trapped charge and flat-band shift, from which the sign of the charge can be determined. To our knowledge, no comparative studies of the trap density at the semiconductor/dielectric interface in a polymer FET based on the subthreshold swing method and MIS capacitance technique have been reported.

Herein, we utilize the mixed-solvent method to produce a P3HT-top/PMMA-bottom (P3HT/PMMA) bilayer for FETs, in which both polymers have been extensively studied and are commercially available. Polymer FETs based on these two materials usually adopt a top-gate/bottom-contact (TGBC) configuration $^{4,5,5,21-25}$ due to P3HT solvents being able to dissolve PMMA. A mixture of a perfect orthogonal solvent (cyclohexane) for PMMA and a good solvent (chloroform) for P3HT was used as the solvent for P3HT. The effect of solvent ratio (degree of orthogonality) on the surface morphologies of the PMMA and P3HT layers, and the electrical properties of the PMMA layers, have been investigated. We have produced P3HT/PMMA bilayers with a clear and lowroughness interface. FETs based on the optimal P3HT/PMMA bilayers with a BGTC configuration and relatively long channel length show good device performance. The trap densities at the P3HT/PMMA interfaces are evaluated from the subthreshold swings and $C-V$ characteristics of the MIS capacitors in the devices and show good agreement.

\section{Experimental}

We selected cyclohexane, a perfect orthogonal solvent of PMMA, ${ }^{26,27}$ and chloroform, a common solvent for P3HT, to prepare a mixed solvent for P3HT. Cyclohexane is also a bad solvent for P3HT, while chloroform is a good solvent for PMMA. The two solvents had suitable boiling points and were miscible. PMMA (molecular weight, 350 000) and P3HT (molecular weight, 130 000; regioregularity, >95\%) were purchased from SigmaAldrich and Rieke Metals, respectively, and used as received.

A schematic of the spin-coating process of P3HT/PMMA bilayers on indium tin oxide (ITO)-coated glass substrates with a sheet resistivity of $15 \Omega \mathrm{sq}^{-1}$ is shown in Fig. 1(a). The substrates were first cleaned with diluted detergent, successively ultrasonicated in deionized water, acetone, and ethyl alcohol for 30 min each, and then dried with nitrogen gas. PMMA was dissolved into ethyl acetate, which is among the best solvents for PMMA, at a concentration of $50 \mathrm{mg} \mathrm{mL}{ }^{-1}$, and stirred for over $12 \mathrm{~h}$. The PMMA films were spin-coated on the precleaned substrates from solution at $1000 \mathrm{rpm}$ for $40 \mathrm{~s}$, and then heated at $150{ }^{\circ} \mathrm{C}$ for $1 \mathrm{~h}$ to allow the solvent to fully evaporate. P3HT was diluted in mixtures of cyclohexane and chloroform with different volume ratios at a concentration of $3 \mathrm{mg} \mathrm{mL}^{-1}$. P3HT layers were deposited on the annealed PMMA films by spin coating the P3HT solutions at $1000 \mathrm{rpm}$ for $40 \mathrm{~s}$, and then dried at $80^{\circ} \mathrm{C}$ for $20 \mathrm{~min}$ to afford the P3HT/PMMA bilayers.

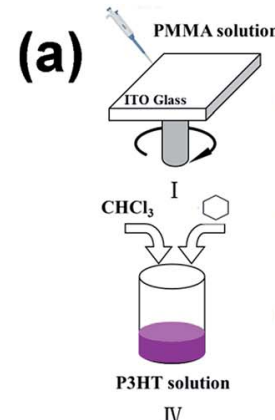

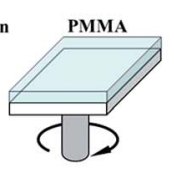

II

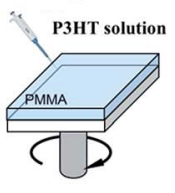

V

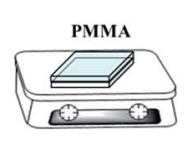

III

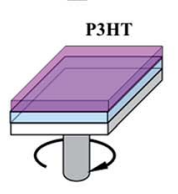

VI (b)

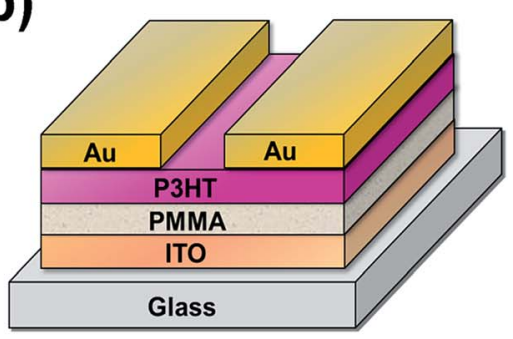

Fig. 1 (a) Schematic illustration of the spin-coating process for P3HT/PMMA bilayers; (b) structure of FETs based on P3HT/PMMA bilayers with a BGTC configuration. 
Fig. 1(b) shows the BGTC structure of the FET devices based on the P3HT/PMMA bilayers. The devices were constructed on ITO-coated glass substrates with ITO, PMMA, and P3HT acting as the gate electrode, gate dielectric layer, and active layer, respectively. $\mathrm{Au}$ films (thickness, $80 \mathrm{~nm}$ ) were thermally deposited on P3HT with a shadow mask to prepare the source and drain electrodes, defining a channel with a relatively long length of $400 \mu \mathrm{m}$ and width of $10000 \mu \mathrm{m}$. Furthermore, a metal-insulator-metal (MIM) structure was used to study the electrical characteristics of the PMMA films, whose surfaces were treated with mixed cyclohexane/chloroform solvents with volume ratios (Cy: $\mathrm{Ch}$ ) of $9: 1,8: 2,7: 3$, and $6: 4$, respectively. The films were sandwiched between ITO-coated glass and thermally evaporated aluminum (Al) films, which served as negative and positive electrodes, to form an ITO/PMMA/Al structure with an area of $4.5 \mathrm{~mm}^{2}$.

The morphologies of the PMMA films and P3HT/PMMA bilayers were characterized by scanning electron microscopy (SEM), and the surface roughness values of the PMMA films were estimated using atomic force microscopy (AFM). The currents of the PMMA dielectric layers and the output and transfer characteristics of the FET devices were measured with a Keithley 4200 semiconductor analyzer. $C-V$ measurements of the PMMA layers and P3HT/PMMA FET devices were conducted with an Agilent E4990A impedance analyzer. All measurements were performed under ambient conditions.

\section{Results and discussion}

\section{Morphologies of polymer semiconductor/dielectric bilayers}

Determining the mixed solvent ratio that provided the necessary degree of orthogonality to PMMA was of vital importance for creating high-quality polymer bilayers. Firstly, the effect of the solvent ratio on P3HT/PMMA bilayer formation and the
P3HT surface morphology was investigated. Fig. 2 shows crosssectional SEM images of the P3HT/PMMA bilayers. The P3HT films were spin-coated on PMMA from the mixed solvents with Cy: Ch ratios of $9: 1,8: 2,7: 3$, and $6: 4$. P3HT/PMMA bilayers were observed to form as the $\mathrm{Cy}: \mathrm{Ch}$ ratio changed from $9: 1$ to $6: 4$, with a clear interface observed at a ratio of $7: 3$. This was due to no chemical reactions occurring between P3HT and PMMA, as indicated by Fourier transform infrared (FTIR) spectra of the blends of the two polymers, ${ }^{28}$ and P3HT having a smaller molecular weight. However, a continuous and uniform P3HT film could not be produced on PMMA (not shown) when the $\mathrm{Cy}: \mathrm{Ch}$ ratio was $5: 5$ because the larger proportion of chloroform resulted in dissolution and severe damage of the PMMA layer.

Cross-sectional SEM images of the bilayers showed that the thickness of the PMMA layer decreased with increasing chloroform concentration in the mixed solvent. The thicknesses of the PMMA layers prepared at $\mathrm{Cy}: \mathrm{Ch}$ ratios of $9: 1,8: 2,7: 3$, and $6: 4$ were estimated to be $420,400,380$, and $240 \mathrm{~nm}$, respectively. This observation was due to more PMMA being dissolved and re-spin-coated on P3HT, leading to a reduction in PMMA thickness due to a lack of strong interactions between P3HT and PMMA. In contrast, the P3HT layer thickness increased with chloroform content. At a $\mathrm{Cy}: \mathrm{Ch}$ ratio of $9: 1$, the P3HT layer was much thinner because it was not completely dissolved in the mixed solvent according to the solubility of $\mathrm{P} 3 \mathrm{HT}$ in chloroform. ${ }^{29}$ As the chloroform ratio was increased to 7 : 3 (30\%), the thickness of P3HT increased to about $100 \mathrm{~nm}$ as P3HT became completely soluble.

The insets of Fig. 2 show top-view SEM images of the bilayers. The solvent ratio was also observed to affect the surface morphology of the P3HT film indirectly. A smooth and uniform P3HT surface was observed when the $\mathrm{Cy}: \mathrm{Ch}$ ratio was varied from $9: 1$ to $7: 3$, whereas some clusters were observed at a $6: 4$
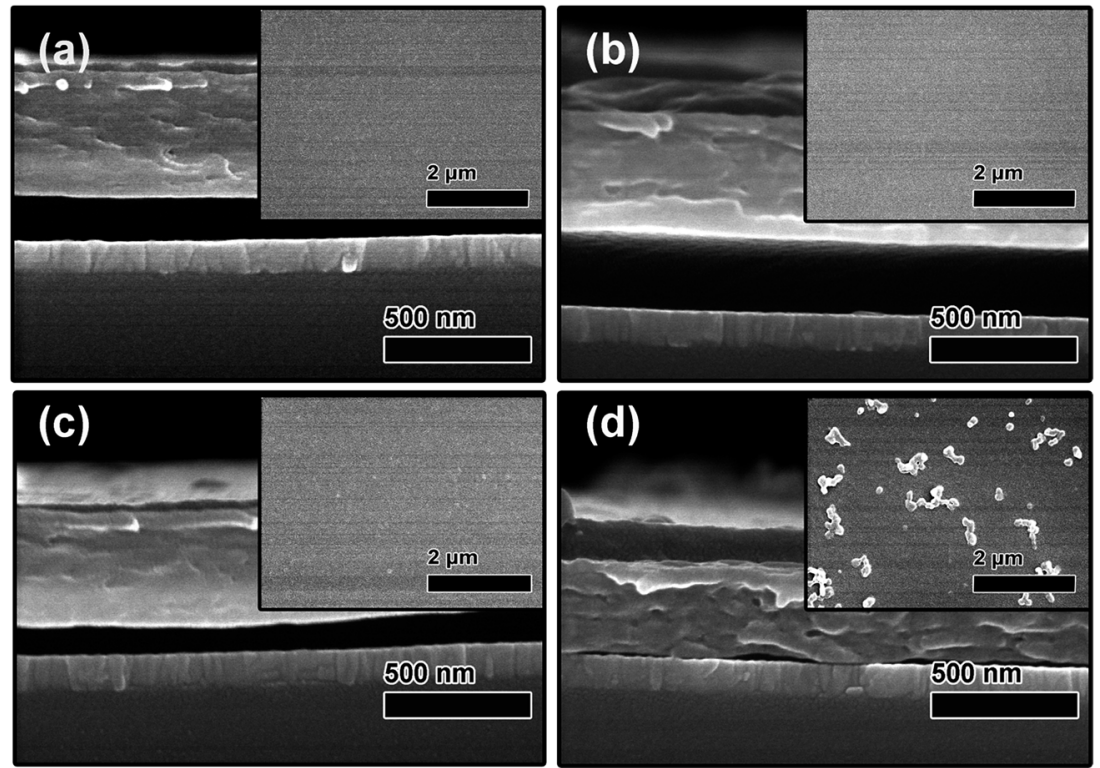

Fig. 2 Cross-sectional and top-view (insets) SEM images of P3HT/PMMA bilayers with P3HT layers spun cast from cyclohexane/chloroform mixed solvents at volume ratios of (a) $9: 1$, (b) $8: 2$, (c) $7: 3$, and (d) $6: 4$. 
ratio. This might be due to increased interface roughness caused by the dissolution of more PMMA and subsequent redeposition of the dissolved PMMA. This is expected when a thin liquid film is deposited onto a rough solid substrate ${ }^{\mathbf{1 0}}$ and will be confirmed by AFM characterization.

In FETs with a BGTC configuration, a dielectric surface was extremely important because a rough surface prevents charge transport by acting as a physical trap and transport barrier, or by disturbing the morphology and the microstructures of the semiconducting layer. ${ }^{30,31}$ To understand the impact of the cyclohexane/chloroform ratio (degree of orthogonality) in the mixed solvent, we characterized the PMMA surface treated with mixed solvent containing no P3HT using AFM measurements. This strategy was feasible because the amount of P3HT in the mixed solution was low and P3HT has no chemical interactions with PMMA. A similar approach ${ }^{8}$ has been used to examine the effects of solvents for PMMA layers on the surfaces of P3HT and poly ([N,N-9-bis(2-octyldodecyl)-naphthalene-1,4,5,8-bis(dicarboximide)-2,6-diyl]-alt-5,59-(2,29-bithiophene)) (P(NDI2ODT2)) films in devices with a top-gate architecture.

Fig. 3 shows AFM micrographs of the pristine PMMA film surface and PMMA film surfaces treated with mixed solvent with $\mathrm{Cy}: \mathrm{Ch}$ ratios of $7: 3$ and $6: 4$, and cyclohexane alone. Both PMMA films spun cast from cyclohexane and the mixed solvent with $7: 3$ ratio exhibited similar surface morphologies to the pristine PMMA film, while the film treated with a $6: 4$ solvent ratio showed some clusters. The root-mean-square (RMS) roughness values were evaluated as 0.391, 0.458, 0.418, and $14.858 \mathrm{~nm}$, respectively. As expected, the RMS roughness of the PMMA film treated with only cyclohexane (100\% Cy) was similar to that of the pristine PMMA film, showing that cyclohexane had no impact on the roughness of PMMA owing to its complete orthogonality to PMMA. The RMS roughness of the PMMA film spun cast from mixed solvent with a $7: 3$ ratio was comparable with that of the pristine PMMA film, which indicated that the PMMA surface remained almost unaffected, even at chloroform concentrations as high as $30 \%$. Nevertheless, when the chloroform ratio was further increased to $6: 4$, the
PMMA film showed a more coarse surface with a high roughness of $14.858 \mathrm{~nm}$, confirming speculation about the increased interface roughness at a solvent ratio of $6: 4$. Evidently, only chloroform altered the degree of orthogonality of the mixed solvent and impacted the PMMA surface quality. When the chloroform ratio reached 6:4 (40\%), a rougher PMMA surface was produced by the dissolution and redeposition of more PMMA during mixed-solvent treatment. The top-view SEM images in the insets of Fig. 2(c) and (d) and the AFM images in Fig. 3(c) and (d) show a similar trend in the surface morphology of the P3HT/PMMA bilayer and the RMS roughness of the mixed-solvent-treated PMMA surface as the chloroform concentration was increased.

Therefore, by simply changing the ratio of the orthogonal solvent to the good solvent, namely the degree of orthogonality with respect to PMMA, we were able to control the interface roughness and produce well-controlled P3HT/PMMA bilayers exhibiting solvent corrosion issues. The critical chloroform content in the mixed solvent was found to be $30 \%$ (Cy: $\mathrm{Ch}=$ $7: 3$ ), below which the RMS roughness of the interface was relatively low, at less than $0.500 \mathrm{~nm}$, and the top P3HT layer showed a smooth and uniform surface. We concluded that the optimal ratio of cyclohexane to chloroform in the mixed solvent for producing a high-quality P3HT/PMMA bilayer was $7: 3$, because the bilayer had a P3HT thickness desirable for FETs, in addition to a clear interface and smooth P3HT surface morphology. Therefore, this mixed-solvent method is not limited to the P3HT/PMMA system, but could also be applied to fabricating other bilayer polymer FETs to overcome solvent corrosion problems encountered in film deposition using a solution-based process.

\section{Electrical properties of polymer dielectric films}

The device performance of the polymer FETs was strongly correlated not only with the semiconductor/dielectric interfaces, but also the electrical properties of gate dielectrics. Fig. 4 shows curves of the current density and capacitance per unit area $v s$. the voltage of the ITO/PMMA/Al devices. The PMMA
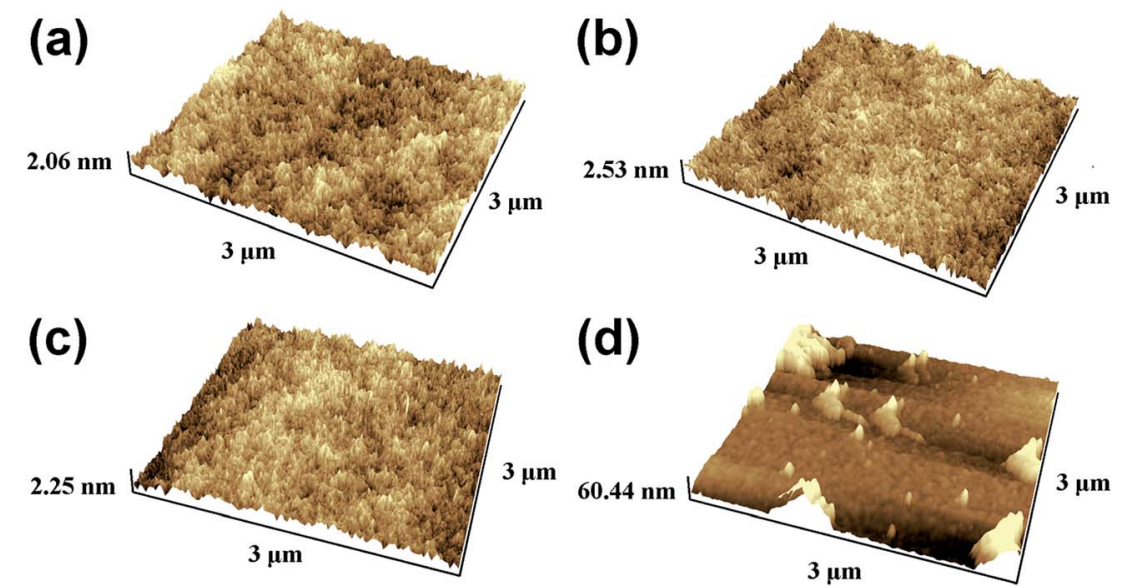

Fig. 3 AFM micrographs $(3 \mu \mathrm{m} \times 3 \mu \mathrm{m})$ of (a) the pristine PMMA film surface and (b) PMMA film surfaces treated with cyclohexane/chloroform mixed solvents with volume ratios of (b) 100\% cyclohexane, (c) $7: 3$, and (d) $6: 4$. 

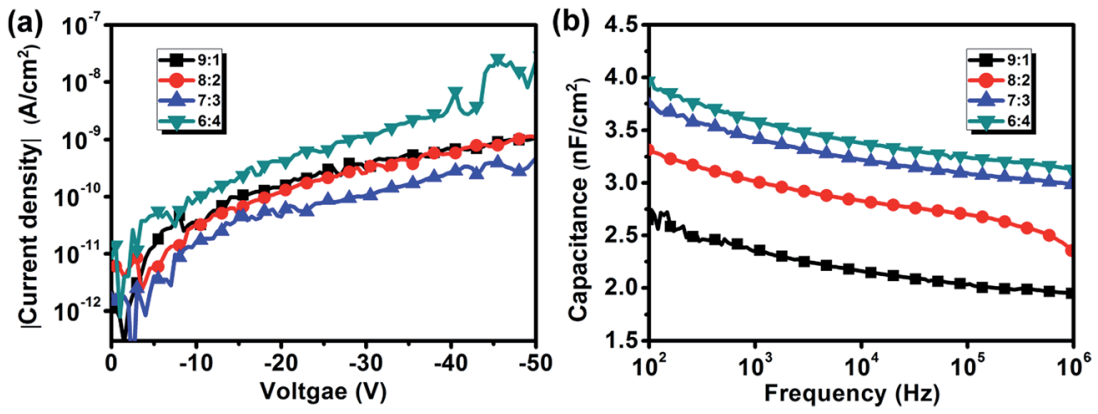

Fig. 4 (a) Current density-voltage and (b) capacitance per unit area-frequency characteristics of PMMA films with surfaces treated with cyclohexane/chloroform mixed solvents at volume ratios of $9: 1,8: 2,7: 3$, and $6: 4$. The PMMA films were sandwiched between ITO and Al electrodes.

surfaces were treated with mixed solvents at $\mathrm{Cy}: \mathrm{Ch}$ ratios of $9: 1,8: 2,7: 3$, and $6: 4$. Fig. 4(a) shows that the current densities of the PMMA films treated with solvents with $9: 1$ and $8: 2$ ratios were similar at voltages ranging from -50 to $0 \mathrm{~V}$. These values were smaller than the current densities of films treated with the $6: 4$ mixed solvent, but larger than those of the films treated with $7: 3$ mixed solvent. The difference in the current density was primarily attributed to the thickness of the PMMA film. The current density increased as the PMMA thickness decreased with increasing chloroform concentration. The current densities for all PMMA films were below $10^{-8} \mathrm{~A} \mathrm{~cm}^{-2}$, indicating that they had good insulating properties. The PMMA film treated with a $7: 3$ solvent ratio possessed the lowest current densities, which guaranteed stable FET operations.

Fig. 4(b) shows that the capacitance per unit area of all ITO/ PMMA/Al devices decreased when the signal frequency was increased from 100 to $10^{6} \mathrm{~Hz}$. This capacitance reduction with increasing frequency was mainly attributed to a reduction in the dielectric constant of PMMA, ${ }^{20}$ which resulted from dipoles failing to reorient with the electric field in the high-frequency range. ${ }^{32}$ The capacitance of the PMMA layer was also observed to increase with increasing chloroform concentration. The capacitance of a dielectric film is known to be inversely proportional to its thickness. Obviously, the increase in capacitance resulted from a reduction in the PMMA thickness with increasing chloroform concentration. The capacitance of the PMMA layer treated with a $7: 3$ solvent ratio at $100 \mathrm{~Hz}$ was $3.75 \mathrm{nF} \mathrm{cm}^{-2}$, which was sufficient for electric charge induction. Evidently, the optimal cyclohexane/chloroform mixed solvent ratio for P3HT was $7: 3$, in which a high-quality P3HT/PMMA bilayer was produced and the treated PMMA layer had a low current density and sufficient capacitance for field-effect conduction.

\section{Electrical performance of polymer bilayer field-effect transistors}

We fabricated polymer FETs based on the P3HT/PMMA bilayers using the optimal cyclohexane/chloroform ratio of $7: 3$, which had an ITO(Gate)/PMMA/P3HT/Au(Source/Drain) structure, as shown in Fig. 1(a). The electrical output and transfer characteristics of the devices are illustrated in Fig. 5. The gate voltage ranged from 0 to $-60 \mathrm{~V}$ in steps of $-10 \mathrm{~V}$ as the source-drain voltage was swept from 0 to $-60 \mathrm{~V}$. The transfer curves shown in Fig. 5(b) were recorded at a source-drain voltage of $-40 \mathrm{~V}$. This device worked through charge accumulation at the P3HT/PMMA interface upon applying a gate voltage and showed a typical p-channel behavior, which consists of linear and saturation regions. The mobility and the threshold voltage can be determined from the transfer characteristics in the saturation regime using eqn (1):

$$
I_{\mathrm{d}}=\frac{W}{2 L} \mu C_{\mathrm{i}}\left(V_{\mathrm{g}}-V_{\mathrm{t}}\right)^{2}
$$

where $W$ and $L$ are the channel width and length, respectively, $\mu$ is the field-effect mobility; $C_{\mathrm{i}}$ is the capacitance per unit area of the gate dielectric, and $V_{\mathrm{t}}$ is the threshold voltage. Analysis of the transfer curve (black) in Fig. 5(b) yielded a hole mobility of $4.4 \times 10^{-3} \mathrm{~cm}^{2} \mathrm{~V}^{-1} \mathrm{~s}^{-1}$ and a threshold voltage of $8.0 \mathrm{~V}$,
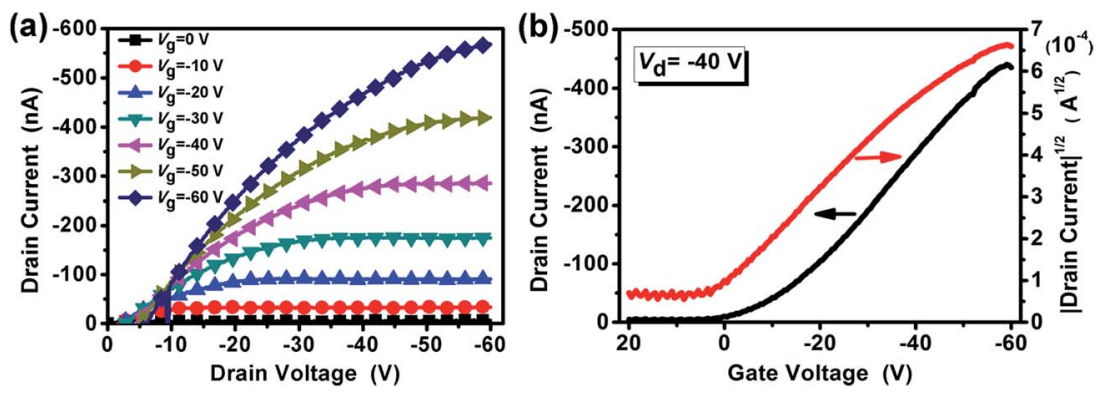

Fig. 5 (a) Output and (b) transfer characteristics of the P3HT/PMMA bilayer FET prepared using cyclohexane/chloroform mixed solvent at the optimal volume ratio of $7: 3$. 
Table 1 Summary of previously reported polymer FETs based on P3HT and PMMA, and our device, indicating structure, fabrication technique, channel length and width, and mobility value

\begin{tabular}{lllll}
\hline Polymer/polymer & Structure & Method & Channel $L / W(\mu \mathrm{m})$ & Mobility $\left(\mathrm{cm}^{2} \mathrm{~V}^{-1} \mathrm{~s}^{-1}\right)$ \\
\hline PMMA/P3HT & TGBC & Spin coating & $100 / 17200$ & $9.99 \times 10^{-3}$ \\
PMMA/P3HT & TGBC & Spin coating & $20 / 1000$ & $0.02-0.04$ \\
PMMA/P3HT & TGBC & Spin coating & $20 / 1000$ & $0.08-0.2$ \\
PMMA/P3HT & TGBC & Spin coating & $50 / 150$ & $2.5 \times 10^{-3}$ \\
PMMA/P3HT & TGBC & Spin coating & $40 / 5000$ & 0.05 \\
PMMA/P3HT & TGBC & Dry transfer & $30 / 110$ & 0.013 \\
P3HT/PMMA & BGTC & Dry transfer & $120 / 3000$ & $1.4 \times 10^{-3}$ \\
P3HT : PMMA & BGTC & Vertical phase separation & - & $0.001-0.022$ \\
P3HT/PMMA & BGTC & Mixed-solvent method & $400 / 10000$ & $4.4 \times 10^{-3}$
\end{tabular}

respectively, which were averaged over 20 devices with standard deviations of $\pm 0.2 \times 10^{-3} \mathrm{~cm}^{2} \mathrm{~V}^{-1} \mathrm{~s}^{-1}$ and $\pm 0.8 \mathrm{~V}$.

For comparison, previously reported polymer FETs based on P3HT and PMMA ${ }^{5,9,11,12,21,22,24,33}$ and our device are summarized in Table 1, indicating structure, fabrication technique, channel length and width, and mobility value. The mobilities of the polymer FETs based on P3HT and PMMA varied over three orders of magnitude, from $10^{-3}$ to $10^{-1} \mathrm{~cm}^{2} \mathrm{~V}^{-1} \mathrm{~s}^{-1}$, depending on device configurations, fabrication methods, and channel lengths. The mobility has been demonstrated to increase as the channel length is reduced. ${ }^{34}$ The highest mobility value of 0.2 $\mathrm{cm}^{2} \mathrm{~V}^{-1} \mathrm{~s}^{-1}$ was achieved in the TGBC devices by spin coating with a channel length of $20 \mu \mathrm{m} .{ }^{24}$ The BGTC devices of a $120 \mu \mathrm{m}$ long channel constructed by a dry transfer technique ${ }^{11}$ had a mobility of $1.4 \times 10^{-3} \mathrm{~cm}^{2} \mathrm{~V}^{-1} \mathrm{~s}^{-1}$. Mobilities ranging from 0.001-0.022 $\mathrm{cm}^{2} \mathrm{~V}^{-1} \mathrm{~s}^{-1}$ were achieved in BGTC devices fabricated by vertical phase separation. ${ }^{12}$ Therefore, despite a much longer channel length of $400 \mu \mathrm{m}$, our FET devices based on P3HT/PMMA bilayers prepared using a cyclohexane-chloroform mixed solvent at the optimal ratio of $7: 3$ exhibited good device performance.

Subthreshold swing $S$ is another important device parameter for quantifying how sharply a transistor can be switched from the off-state to the on-state by a gate voltage. ${ }^{17-19} S$ is defined as the change in the gate voltage needed to induce a drain-current change of one order of magnitude (eqn (2)):

$$
S=\frac{\mathrm{d} V_{\mathrm{g}}}{\mathrm{d}\left(\log I_{\mathrm{d}}\right)} .
$$

Fig. 6(a) shows the same data as Fig. 5(b), but on a logarithmic scale. The red line is a linear fit of the measured data in the voltage region from the off-state to the on-state, from which a subthreshold swing $(S)$ of $14 \mathrm{~V}$ per decade (dec) was estimated for the P3HT/PMMA FET device. This value was within the range of the subthreshold swings $\left(1-20 \mathrm{~V} \mathrm{dec}^{-1}\right)$ reported for organic and polymer FETs in most published studies. ${ }^{35}$

\section{Comparative studies of traps at polymer semiconductor/ dielectric interfaces}

The quality of the semiconductor/dielectric interface in a polymer FET determines the subthreshold swing. ${ }^{17}$ With a simplistic assumption that the density of interface traps is independent of energy, the maximum interface trap density is given by eqn (3):

$$
N_{\mathrm{it}}^{\max }=\left[\frac{S \log e}{k T / q}-1\right] \frac{C_{\mathrm{i}}}{q},
$$

where $k$ is the Boltzmann constant, $q$ is the elementary charge, $T$ is the absolute temperature, and $C_{\mathrm{i}}$ is the capacitance per unit area of the dielectric. The maximum density of the interface traps of our device was derived to be $3.6 \times 10^{12} \mathrm{~cm}^{-2} \mathrm{eV}^{-1}$, with $S=14 \mathrm{~V} \mathrm{dec}^{-1}$ and $C_{\mathrm{i}}=2.43 \mathrm{nF} \mathrm{cm}{ }^{-2}$. This value was moderate compared with the maximum interface trap densities of $10^{10}$ to $10^{13} \mathrm{~cm}^{-2} \mathrm{eV}^{-1}$ published for polymer FETs, ${ }^{18,36,37}$ suggesting that the device with P3HT prepared from the mixed solution of cyclohexane and chloroform at the optimal ratio of $7: 3$ has a good P3HT/PMMA interface.

Lastly, to further study the properties of the traps at the P3HT/PMMA interface in the FET device, we conducted highfrequency capacitance measurements ${ }^{19}$ directly on the device with a voltage only applied between the ITO gate electrode and one Au electrode, which apparently constructed an ITO/PMMA/ P3HT MIS structure. The area of the MIS capacitor was 0.2 $\mathrm{cm}^{-2}$, defined by the area of the Au electrode. The capacitance of the FET device was measured by sweeping the bias voltage from -40 to $+40 \mathrm{~V}$ with a small superimposed AC signal of $100 \mathrm{mV}$ at $500 \mathrm{kHz}$ on top of a preselected DC voltage. Fig. 6(b) shows typical curves of capacitance per unit area $(C) v s$. voltage for the MIS capacitor from its normalized value $\left(C / C_{0}\right)$ to the maximum value $\left(C_{0}\right)$ at $-40 \mathrm{~V}$. These $C-V$ curves were similar to that of an ideal MIS structure. ${ }^{19}$ Accumulation, transition, and full depletion regions, but no inversion region, were clearly observed as the voltage ranged from -40 to $40 \mathrm{~V}$, which was consistent with the capacitance $v s$. voltage behavior of polymer MIS structures. ${ }^{20,38}$ The maximum value of the capacitance per unit area at $-40 \mathrm{~V}$ was equal to that of the PMMA insulating layer $\left(C_{\mathrm{i}}\right)$ because the P3HT semiconducting layer driven into accumulation was in a conducting state.

The difference in work function between ITO $(5.1 \mathrm{eV})$ and P3HT $(5.0 \mathrm{eV})$ was $0.1 \mathrm{eV}$ in the P3HT/PMMA FET device and could be considered negligible. ${ }^{20,38}$ We evaluated the trap density at the P3HT/PMMA interface according to deviations in the $C-V$ curve from that of an ideal MIS capacitor, if all charges in the dielectric were assumed to be located at the interface. 

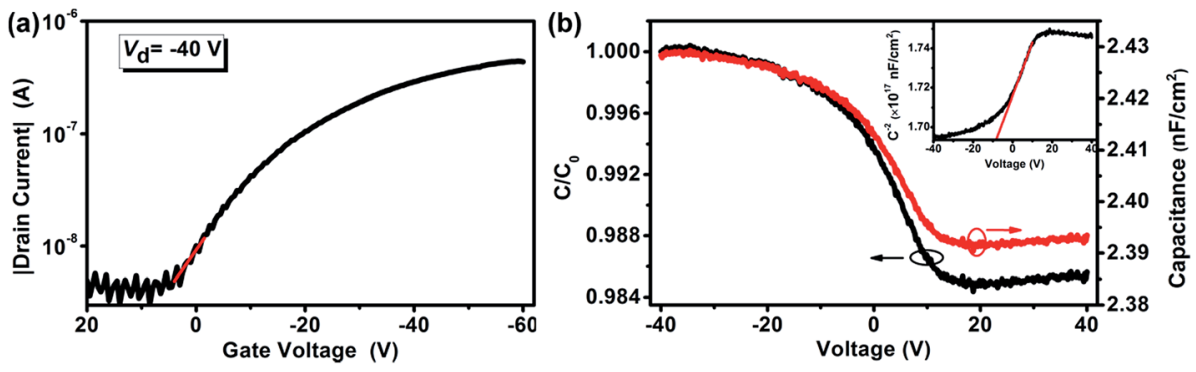

Fig. 6 (a) Logarithmic plot of the data shown for P3HT/PMMA FET in Fig. 5(b). (b) Capacitance per unit area-voltage characteristics at a frequency of $500 \mathrm{kHz}$ and normalized capacitance with respect to the maximum capacitance $C_{0}$ in accumulation at $-40 \mathrm{~V}$ for the polymer FET based on the P3HT/PMMA bilayer prepared with cyclohexane-chloroform mixed solvent at the optimal volume ratio of $7: 3$. The voltage was only applied to the ITO gate electrode and one Au electrode. Inset: Mott-Schottky plot of capacitance vs. voltage.

This assumption was reasonable because a greater effect on the $C-V$ behavior was expected if the charges in the dielectric were closer to the interface. In general, charges in the interface traps of polymer MIS capacitors can shift the flat band voltage, which is zero for an ideal MIS capacitor, and deform the $C-V$ curves. ${ }^{20,38}$ The total capacitance under flat-band conditions ${ }^{19}$ for an ideal MIS structure can be expressed as follows (eqn (4)):

$$
C_{\mathrm{FB}}=\frac{\varepsilon_{0} \varepsilon_{\mathrm{i}} \varepsilon_{\mathrm{s}}}{\varepsilon_{\mathrm{s}} d_{\mathrm{i}}+\varepsilon_{\mathrm{i}} \sqrt{K T \varepsilon_{0} \varepsilon_{\mathrm{s}} / N_{\mathrm{A}} q^{2}}}
$$

where $\varepsilon_{0}$ is the vacuum permittivity, $\varepsilon_{\mathrm{i}}$ and $\varepsilon_{\mathrm{S}}$ are the relative dielectric constants of the dielectric and semiconductor, respectively, $d_{\mathrm{i}}$ is the thickness of the dielectric, and $N_{\mathrm{A}}$ is the dopant density of the semiconductor.

Usually, the dopants in a semiconducting polymer are residual or unintentional impurities. The dopant density can be extracted based on the standard Mott-Schottky relation for an MIS capacitor in depletion (eqn (5)): $:^{39-41}$

$$
\frac{\partial}{\partial V}\left(C^{-2}\right)=\frac{2}{q \varepsilon_{\mathrm{s}} N_{\mathrm{A}} A^{2}},
$$

where $C$ is the total capacitance of the device. The inset of Fig. 6(b) shows the Mott-Schottky plot of $C^{-2} v s$. V. From the linear part of the curve, a residual impurity or dopant density of $2.0 \times 10^{17} \mathrm{~cm}^{-3}$ in P3HT was derived with $\varepsilon_{\mathrm{S}}=3,{ }^{40}$ which agreed with the order of magnitude of $10^{17}$ obtained for P3HT in the previous reports. ${ }^{41,42}$ The relative dielectric constant $\varepsilon_{\mathrm{i}}$ of PMMA is reported to decrease with increasing frequency and is 2.6 at 1 MHz. ${ }^{20}$ The thickness of the PMMA layer was about $370 \mathrm{~nm}$, as estimated from the cross-sectional SEM image of the P3HT/ PMMA bilayer shown in Fig. 2(c). Substituting the values of $N_{\mathrm{A}}, d_{\mathrm{i}}, \varepsilon_{\mathrm{s}}$, and $\varepsilon_{\mathrm{i}}$ into eqn (4) obtained a flat band capacitance of $2.40 \mathrm{nF} \mathrm{cm}{ }^{-2}$ for the device.

The corresponding voltage of this flat band capacitance in Fig. $6(\mathrm{~b})$ was the flat band voltage, $V_{\mathrm{FB}}$, which was about $6 \mathrm{~V}$ for the device. The flat band voltage shift in the positive voltage direction indicated negative charges trapped at the P3HT/ PMMA interface with acceptor-type interface traps. ${ }^{20,38}$ Assuming that all charges in the dielectric were located at the interfaces, the density of the interface traps, $N_{\mathrm{it}}$, responsible for the $V_{\mathrm{FB}}$ shift was calculated using eqn (6): ${ }^{20}$

$$
V_{\mathrm{FB}}=\Phi_{\mathrm{MS}}-\frac{q N_{\mathrm{it}}}{C_{\mathrm{i}}} .
$$

where $\Phi_{\mathrm{MS}}$ is the work function difference between the metal and the semiconductor, which was $0.1 \mathrm{eV}$ for our device. Therefore, the density of the interface traps in the FET device was derived to be $2.3 \times 10^{11} \mathrm{~cm}^{-2}$ using eqn (6), which is in the value range of $10^{11}$ to $10^{12} \mathrm{~cm}^{-2}$ for the PMMA/P3HT interfaces. ${ }^{20}$

Actually, the interface trap density derived from the subthreshold swing was the same as that calculated from the capacitance measurement, but expressed in a different unit. The interface trap density in $\mathrm{cm}^{-2} \mathrm{eV}^{-1}$ characterized the distribution of the interface trap levels across the energy bandgap ${ }^{19}$ between LUMO and HOMO levels in a polymer semiconductor. Therefore, it was reasonable that the interface trap density of the PMMA/P3HT FET device in $\mathrm{cm}^{-2} \mathrm{eV}^{-1}$ evaluated from the subthreshold swing was larger than that in $\mathrm{cm}^{-2}$ estimated from the $C-V$ measurements.

\section{Conclusion}

We have proposed a mixed-solvent method for producing highquality polymer bilayers by spin-coating to solve solvent corrosion issues at the interfaces. This method has been applied to fabricating P3HT/PMMA bilayers. The mixed solvent for P3HT consisted of cyclohexane, an orthogonal solvent of PMMA, and chloroform, a good solvent of P3HT. By changing the ratio of cyclohexane to chloroform, namely the degree of orthogonality with respect to PMMA, we produced well-controlled P3HT/ PMMA bilayers. The optimal cyclohexane-chloroform ratio for preparing a high-quality P3HT/PMMA bilayer for field-effect conduction was found to be $7: 3$. The bilayer under these conditions had a clear interface with low roughness, a smooth P3HT surface morphology, a desirable P3HT thickness, and a PMMA dielectric layer with good electric properties.

We investigated the performance of FET devices based on the optimal P3HT/PMMA bilayers with a BGTC configuration. The device exhibited a comparable hole mobility of $4.4 \times 10^{-3}$ $\mathrm{cm}^{2} \mathrm{~V}^{-1} \mathrm{~s}^{-1}$ despite a much longer channel length of $400 \mu \mathrm{m}$. The subthreshold swing of the device was $14 \mathrm{~V} \mathrm{dec}^{-1}$, from which the maximum density of the interface traps was 
determined to be $3.6 \times 10^{12} \mathrm{~cm}^{-2} \mathrm{eV}^{-1}$, characterizing the distribution of the interface trap levels across the bandgap between the LUMO and HOMO levels in P3HT. The results of $C$ $V$ measurements on the MIS structure in the device suggested that the traps at the P3HT/PMMA interface were acceptor-type and that the density of the interface traps was $2.3 \times 10^{11}$ $\mathrm{cm}^{-2}$. Notably, the interface trap density in $\mathrm{cm}^{-2} \mathrm{eV}^{-1}$, evaluated from the subthreshold swing, and that in $\mathrm{cm}^{-2}$, estimated from the $C-V$ measurements, were in good agreement. Our mixed-solvent method is not limited to the P3HT/PMMA system, but could also be applied to fabricating other polymer FETs based on various combinations of polymer semiconductors and dielectrics that show solvent corrosion problems during successive film deposition using a solution-based process.

\section{Conflicts of interest}

There are no conflicts to declare.

\section{Acknowledgements}

This work was supported by the National Natural Science Foundation of China (No. 61675018, 61674012, 61475014, and 61775011).

\section{References}

1 X. Sun, C.-a. Di and Y. Liu, J. Mater. Chem., 2010, 20, 2599.

2 T. G. Bäcklund, H. G. O. Sandberg, R. Österbacka, H. Stubb,

T. Mäkelä and S. Jussila, Synth. Met., 2005, 148, 87-91.

3 A. B. Rodríguez, M. R. Tomlinson, S. Khodabakhsh, J.-F. Chang, F. Cousin, D. Lott, H. Sirringhaus, W. T. S. Huck, A. M. Higgins and M. Geoghegan, J. Mater. Chem. C, 2013, 1, 7736.

4 Y. Yan, L. B. Huang, Y. Zhou, S. T. Han, L. Zhou, J. Zhuang, Z. X. Xu and V. A. Roy, Sci. Rep., 2015, 5, 15770.

5 L. Zhang, D. Yang, S. Yang and B. Zou, Appl. Phys. A: Mater. Sci. Process., 2014, 116, 1511-1516.

6 C. de Col, A. Nawaz, I. Cruz-Cruz, A. Kumar, A. Kumar and I. A. Hümmelgen, Org. Electron., 2015, 17, 22-27.

7 K.-J. Baeg, A. Facchetti and Y.-Y. Noh, J. Mater. Chem., 2012, 22, 21138.

8 L. S. Cardoso, J. C. Stefanelo and R. M. Faria, Synth. Met., 2016, 220, 286-291.

9 K.-J. Baeg, ETRI J., 2011, 33, 887-896.

10 S. S. Chang, A. B. Rodríguez, A. M. Higgins, C. Liu, M. Geoghegan, H. Sirringhaus, F. Cousin, R. M. Dalgleish and Y. Deng, Soft Matter, 2008, 4, 2220.

11 J. Park, S.-O. Shim and H. H. Lee, Appl. Phys. Lett., 2005, 86, 073505.

12 X. Wang, W. H. Lee, G. Zhang, X. Wang, B. Kang, H. Lu, L. Qiu and K. Cho, J. Mater. Chem. C, 2013, 1, 3989.

13 K. P. Pernstich, D. Oberhoff, C. Goldmann and B. Batlogg, Appl. Phys. Lett., 2006, 89, 213509.

14 H. L. Gomes, P. Stallinga, M. Cölle, D. M. de Leeuw and F. Biscarini, Appl. Phys. Lett., 2006, 88, 082101.
15 F.-Y. Yang, K.-J. Chang, M.-Y. Hsu and C.-C. Liu, J. Mater. Chem., 2008, 18, 5927.

16 J. Park, S. Y. Park, S.-O. Shim, H. Kang and H. H. Lee, Appl. Phys. Lett., 2004, 85, 3283-3285.

17 W. L. Kalb and B. Batlogg, Phys. Rev. B: Condens. Matter Mater. Phys., 2010, 81, 035327.

18 K. N. N. Unni, S. Dabos-Seignon and J.-M. Nunzi, J. Mater. Sci., 2006, 41, 317-322.

19 S. M. Sze and k. K. Ng, Physics of Semiconductor Devices, John Wiley \& Sons, 2006.

20 M. Estrada, F. Ulloa, M. Avila, J. Sanchez, A. Cerdeira, A. Castro-Carranza, B. Iniguez, L. F. Marsal and J. Pallares, IEEE Trans. Electron Devices, 2013, 60, 2057-2063.

21 M. Estrada, I. Mejia, A. Cerdeira and B. Iñiguez, Solid-State Electron., 2008, 52, 53-59.

22 I. Mejia, M. Estrada and M. Avila, Microelectron. Reliab., 2008, 48, 1795-1799.

23 J. Li, Z. Sun and F. Yan, Adv. Mater., 2012, 24, 88-93.

24 K.-J. Baeg, D. Khim, D.-Y. Kim, J. B. Koo, I.-K. You, W. S. Choi and Y.-Y. Noh, Thin Solid Films, 2010, 518, 4024-4029.

25 L. Reséndiz, M. Estrada, A. Cerdeira, B. Iñiguez and M. J. Deen, Org. Electron., 2010, 11, 1920-1927.

26 D. Ennis, H. Betz and H. Ade, J. Polym. Sci., Part B: Polym. Phys., 2006, 44, 3234-3244.

27 Y. Ma, X. Cao, X. Feng, Y. Ma and H. Zou, Polymer, 2007, 48, 7455-7460.

28 M. E. Nicho, C. H. García-Escobar, M. C. Arenas, P. AltuzarCoello, R. Cruz-Silva and M. Güizado-Rodríguez, Mater. Sci. Eng., B, 2011, 176, 1393-1400.

29 F. Machui, S. Langner, X. Zhu, S. Abbott and C. J. Brabec, Sol. Energy Mater. Sol. Cells, 2012, 100, 138-146.

30 Y. Don Park, J. A. Lim, H. S. Lee and K. Cho, Mater. Today, 2007, 10, 46-54.

31 Z. Bao and J. Locklin, Organic field-effect transistors, CRC Press, Boca Raton, 2007.

32 E. Reis Simas, E. S. H. Kang, A. Gassmann, E. Katholing, S. Janietz and H. von Seggern, J. Mater. Chem. C, 2015, 3, 9217-9223.

33 M. Wang, F. Jakubka, F. Gannott, M. Schweiger and J. Zaumseil, Org. Electron., 2014, 15, 809-817.

34 G. Wang, J. Swensen, D. Moses and A. J. Heeger, J. Appl. Phys., 2003, 93, 6137-6141.

35 O. Marinov, M. J. Deen and B. Iniguez, IEE P.-Circ. Dev. Syst., 2005, 152, 189.

36 D. M. Taylor and N. Alves, J. Appl. Phys., 2008, 103, 054509.

37 I. Torres, D. M. Taylor and E. Itoh, Appl. Phys. Lett., 2004, 85, 314-316.

38 Y. K. Lee and S. P. Murarka, Mater. Res. Bull., 1999, 34, 869876.

39 E. J. Meijer, A. V. G. Mangnus, C. M. Hart, D. M. de Leeuw and T. M. Klapwijk, Appl. Phys. Lett., 2001, 78, 3902-3904.

40 O. Marinov, M. J. Deen, B. Iniguez and B. Ong, J. Vac. Sci. Technol., A, 2006, 24, 649-653.

41 I. Torres and D. M. Taylor, J. Appl. Phys., 2005, 98, 073710.

42 D. M. Taylor, J. A. Drysdale, I. Torres and O. Fernández, Appl. Phys. Lett., 2006, 89, 183512. 